Dr. A. Skeet, 2A Sydervelt Road, CANVEY ISLAND, Essex SS8 9SU

Dr. G. C. Smith, Chevington House, BOURNE, Lincolnshire

Mr. P. Squire, MA, 50 Redford Road, Colinton, EDINBURGH EH13 OAC

Mr. P. G. Stone, DPE, 24 Brookside Road, LOUGHBOROUGH, Leicesterhsire LE11 3PO

Miss Sheila Stuart, MSRG, 29 Manor Road, Wighill Lane, TADCASTER, Near York

Dr. D. J. Thomas, 22 Bowham Avenue, BRIDGEND, Mid-Glamorgan

Dr. P. van Beek, Harderwijkerweg 34, DIEREN, Netherlands

Dr. Rita Vanwollenghem, Sinte Walburgastraat 20, BRUGGE, Belgium

Dr. A. K. Varma, 11A Rajendranagar, PATNA-800016, Bihar, India

Dr. J. T. Woodings, 56 Leicester Road, UPPINGHAM, Rutland, Near Leics.

Dr. Mabel Yates, 5 St. Catharine's Walk, LEEDS LS8

Dr. R. Yeo Ngo Tee, 495 1\&/1\& Road, Petaling Jaya, SELANGOR, Malaysia

\section{STUDENT MEMBERS}

Mr. H. M. Alba, Via Bonavia 12, 40068 San Lazzaro di Savena, BOLOGNA, Italy

Mr. M. A. Al-Dawalibi, 20 Buchanan Street, Swinton, MANCHESTER

Miss M. L. Curtis, School of Physiotherapy, Orthopaedic Hospital, OSWESTRY,

Shropshire SY10 7AG

Mr. D. R. Gorman, 73 Balmoral Avenue, BELFAST BT9 6NY

Mr. P. Harrington, 12 Bowood Street, LIVERPOOL L8

Miss G. L. Rosser, 18 Chatham Grove, Withington, MANCHESTER

Mr. S. N. Saddler, 324 Oxgangs Road North, EDINBURGH EH13 9NE

Mr. E. M. Sheehy, 18 High Street, Quorn, LOUGHBOROUGH, Leics.

Mr. R. M. Tripp, 126 Grange Road, Soothill, BATLEY, West Yorkshire

Mr. J. W. Whiteney, 38 Bishopstrow, WARMINSTER, Wiltshire
H. Robson

J. Williams

H. Robson

H. Robson

F. Collison

R. Archibald

H. Robson

H. Robson

H. Robson

H. Robson

H. Robson

H. Robson

H. Robson

D. Masterson

H. Robson

H. Robson

V. Thomas

H. Robson

D. Brodig o

E. Hamleq

F. Collisore

B. Davies

Erratum: In Vol. 12 No. 1. Brit.J.Sports Med. p. 61, the new members listed were ELECTED on January 25th, 1978, and not PROPOSED FOR ELECTION. - Ed. BJSM. 